\title{
Near-field photoluminescence imaging of single defects in a ZnSe quantum-well structure at low temperatures
}

\author{
G. von Freymann, D. Lüerßen, C. Rabenstein, M. Mikolaiczyk, H. Richter, H. Kalt, \\ Th. Schimmel, and M. Wegener ${ }^{\mathrm{a})}$ \\ Institut für Angewandte Physik, Universität Karlsruhe (TH), D-76128 Karlsruhe, Germany
}

K. Okhawa and D. Hommel

Institut für Festkörperphysik, Universität Bremen, D-28334 Bremen, Germany

(Received 10 August 1999; accepted for publication 12 November 1999)

\begin{abstract}
Single bright-spot, bow-tie-shaped defects in a $\mathrm{ZnSe}$ heterostructure are spectroscopically imaged with $200 \mathrm{~nm}$ spatial resolution at a sample temperature of $20 \mathrm{~K}$. The local thickness variations in the single ZnSe quantum-well lead to a lateral double-well potential resulting in a shift and enhancement of the quantum-well photoluminescence. The luminescence images of the $\mathrm{ZnMgSSe}$ barrier material are governed by nonradiative recombination at the four associated Frank partial dislocations. (C) 2000 American Institute of Physics. [S0003-6951(00)01702-2]
\end{abstract}

Defects in semiconductor heterostructures are usually undesired and often thought of solely as centers of nonradiative carrier recombination in the quantum wells. In this context, it did come as a surprise to us when Ref. 1 recently observed that pairs of stacking faults ${ }^{2,3}$ bounded by Frank partial dislocations in $\mathrm{ZnSe}$ quantum-well structures showed up as pairs of bright spots in microphotoluminescence images. ${ }^{1}$ Moreover, it was shown by electron microscopy ${ }^{1}$ that these bow-tie-shaped defects are very closely identical in shape, size, and orientation with respect to the crystallographic axes. The aim of the present work is to clarify the nature of the resulting potential landscape in the quantum
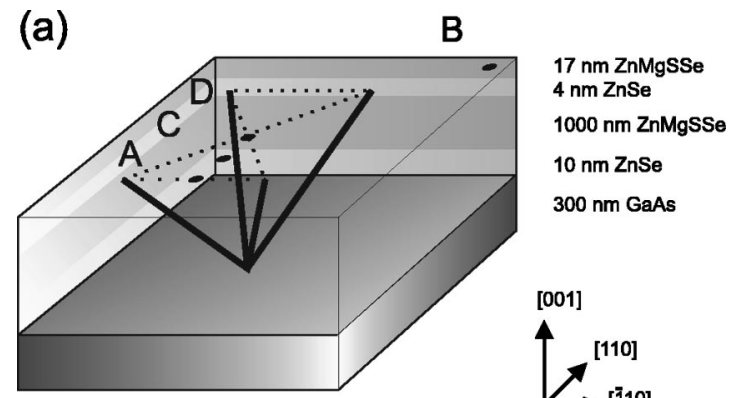

$10 \mathrm{~nm} \mathrm{ZnSe}$

$300 \mathrm{~nm}$ GaAs

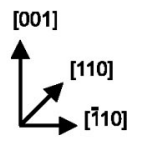

(b)

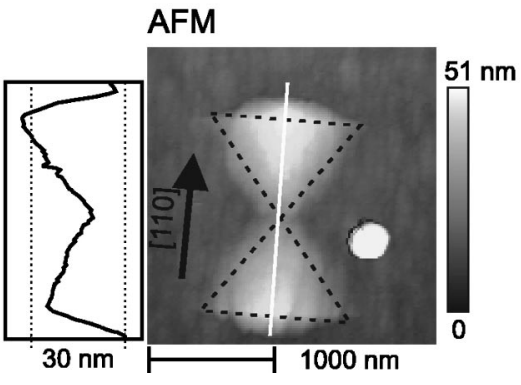

FIG. 1. (a) Scheme of the sample-layer sequence and the bow-tie structures related to pairs of stacking faults bounded by Frank partial dislocations. (b) A topographic image taken with an atomic force microscope (AFM) indicates a surface topography with a height of about $30 \mathrm{~nm}$.

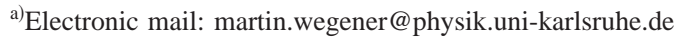

well and the role of nonradiative carrier recombination in the wells and the barriers by scanning near-field optical spectroscopy at low temperatures.

The sample investigated here was grown by molecularbeam epitaxy on a 300-nm-thick GaAs buffer on a GaAs (001) substrate (Fig. 1). This sample contains a single 4-nm-

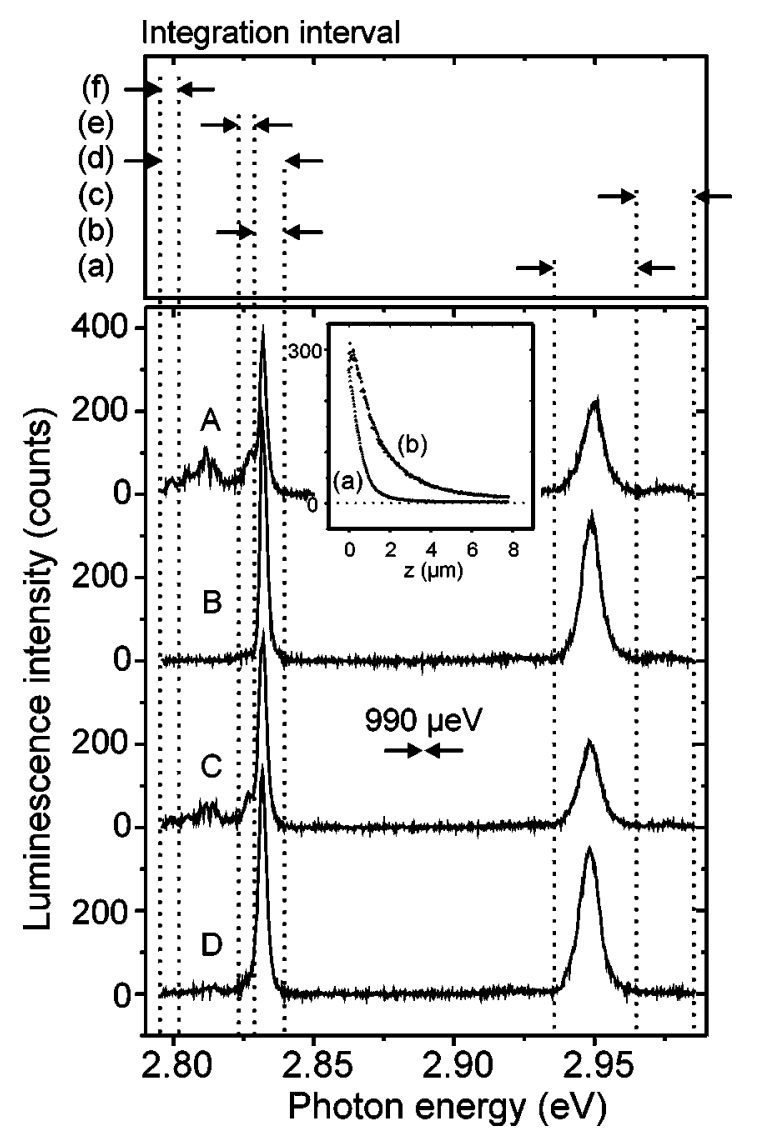

FIG. 2. Photoluminescence spectra taken at four different spatial positions (A, B, C, and D) as defined in Fig. 1. The sample temperature is $T$ $=20 \mathrm{~K}$, excitation with $300 \mathrm{nW}$ power of 400-nm-wavelength light. The upper part defines spectral intervals (a)-(f), which correspond to the images shown in Figs. 3(a)-3(f). The inset shows an approach curve for the intervals (a) and (b). 
(a)

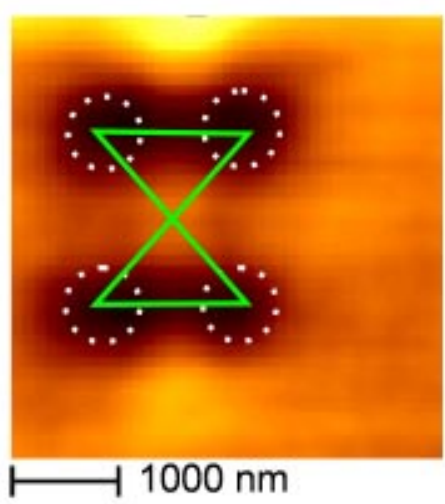

(b)

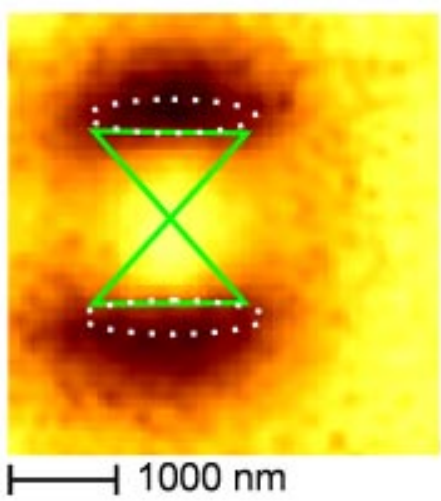

(c)

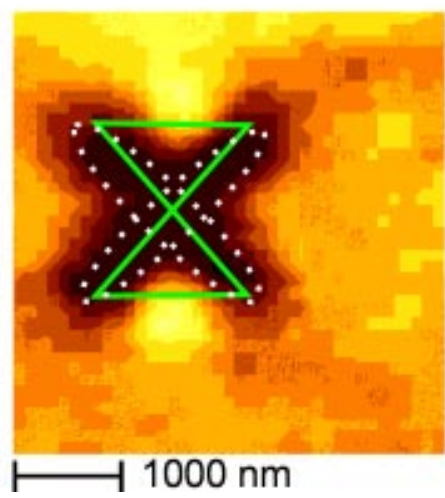

39364

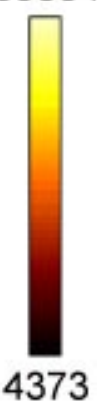

13348

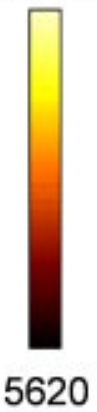

1507

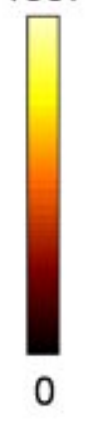

(d)

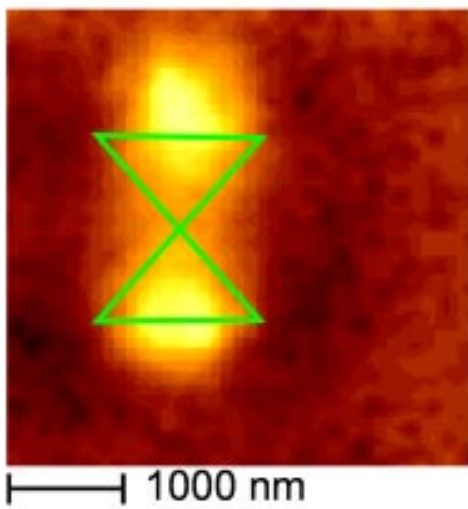

21946

(e)

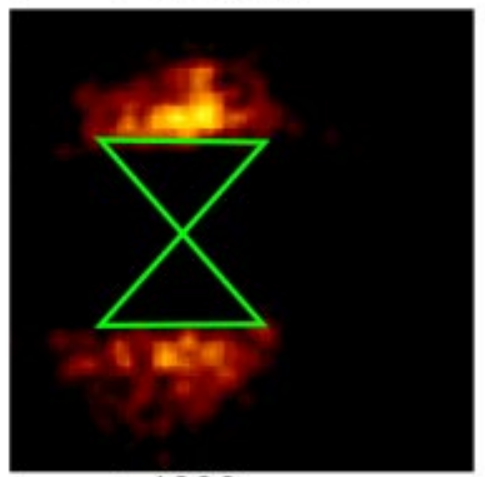

572

(f)
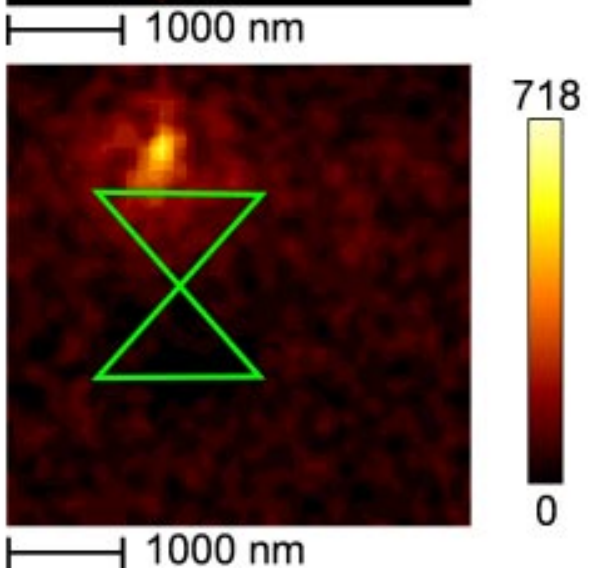

FIG. 3. (Color) Photoluminescence false color images corresponding to the photon energy intervals (a)-(f) defined at the top of Fig. 2. The sample temperature is $T=20 \mathrm{~K}$, excitation with $300 \mathrm{nW}$ power of 400-nm-wavelength light. For clarity, the bow-tie defined in Fig. 1 is shown in each image by the green lines. The dotted lines are guides to the eye. The $1000 \mathrm{~nm}$ bar on the bottom defines the length scale. The individual photoluminescence intensity scales on the right-hand side refer to the integrated number of photon counts in the respective spectral interval.

thin $\mathrm{ZnSe}$ quantum well with quaternary $\mathrm{Zn}_{1-x} \mathrm{Mg}_{x} \mathrm{~S}_{y} \mathrm{Se}_{1-y}$ (ZnMgSSe) barriers with $x \approx 0.1$ and $y \approx 0.1$ on either side. Note that the ZnMgSSe cap layer is $17 \mathrm{~nm}$ thin. Ref. 1 showed that pairs of stacking faults bounded by four Frank partial dislocations originate at the GaAs/ZnSe interface and propagate through the 1000-nm-thick ZnMgSSe barrier layer towards the surface. A bow-tie-shaped distortion of the sample surface is apparent from the topographic image [taken with an atomic-force microscope (AFM)], see Fig. $1(\mathrm{~b})$.

For the optical experiments, we frequency double about $1 \mathrm{~W}$ of power at an $800 \mathrm{~nm}$ wavelength derived from a cw Ti:sapphire laser in a 2-mm-thick BBO crystal to obtain about $3 \mu \mathrm{W}$ of power at a $400 \mathrm{~nm}$ wavelength. $300 \mathrm{nW}$ are effectively coupled into an optical fiber. The light propagates towards a nanometer-scale-apex, which is formed by a twostep selective etching process. Electron micrographs of identical fiber tips have been shown in Ref. 4. We scan this Downloaded 06 Mar 2009 to 129.13.72.198. Redistribution subject to uncoated tip in a constant height of $70 \mathrm{~nm}$ above the highest elevation of the defect (see Fig. 1), i.e., the feedback loop is inactive. The constant height mode is employed because the constant distance mode is expected to be influenced by topography artifacts. The photoluminescence from the sample, which is held at a constant temperature of $T=20 \mathrm{~K}$, is collected by the same uncoated fiber tip (the same technique has been applied to different semiconductor systems at room temperature, see Refs. 5, and 6) and is sent into a grating spectrometer which is connected to a liquid-nitrogen-cooled, back-illuminated charge-coupled-device (CCD) camera. The spectral resolution is better than $1 \mathrm{meV}$ (full width at half maximum) and is indicated in Fig. 2. Typically, a total of 1600 spectra are taken on a $4 \mu \mathrm{m} \times 4 \mu \mathrm{m}$ area with $100 \mathrm{~nm}$ separation between points and with an integration time of 0.5 s per point. We have investigated about 20 different bow-tieshaped defects of this sample in detail. The results shown o AIP license or copyright; see http://apl.aip.org/apl/copyright.jsp 
here for one particular defect are representative for all of them.

Selected spectra for four positions A, B, C, and D within the $x y$ plane (defined in Fig. 1) are depicted in Fig. 2. When positioning the fiber tip above the homogeneous regions of the quantum well (position B), we observe photoluminescence from the quantum well around a photon energy of $\hbar \omega=2.831 \mathrm{eV}$, from the top $\mathrm{ZnMgSSe}$ cap layer around $\hbar \omega=2.949 \mathrm{eV}$, and a much weaker contribution from the lower $\mathrm{ZnMgSSe}$ barrier around $\hbar \omega=2.975 \mathrm{eV}$. The two nominally identical ZnMgSSe epilayers emit at different wavelengths. This is very likely due to slightly different actual compositions. When moving to spots near the bow-tie (see positions A, C, and D), the relative weights of these spectral contributions change. Furthermore, a series of strong additional photoluminescence lines below $2.82 \mathrm{eV}$ photon energy occurs. These reproducible lines are most prominent in $\mathrm{A}$ and $\mathrm{C}$. These lines have previously been seen in microphotoluminescence experiments. ${ }^{1}$ Reference 1 obtained good fits to these lines by a simple model which accounts for a local thickening of the quantum well by an integer number of bilayers. Thus, each photoluminescence peak corresponds to a different local quantum-well thickness. The lowest-energy peaks correspond to a thickening by as much as 12 bilayers. This shows that the thickening of $30 \mathrm{~nm}$ seen in Fig. 1(b) of the 1031-nm-thick epitaxial film is not proportionally distributed over the different layers. Unfortunately, the detailed mechanism of the formation of such defects is presently not understood. Strain effects, on the other hand, are not likely to produce a series of sharp photoluminescence lines. The ZnSe quantum well is homogeneously strained, however, stacking faults are not known to relax strain. A study on the formation of such defects has shown ${ }^{7}$ that the compressive strain of the $\mathrm{ZnSe}$ layers is relaxed around the dislocation lines. Thus, the other parts of the sample remain essentially homogeneously strained.

In order to get an overview of the behavior, we integrate the measured spectra over various intervals in photon energy and plot the resulting real-space images. The images (a)-(f) in Fig. 3 correspond to the intervals (a)-(f) defined at the top of Fig. 2. Figure 3(a) images the thin cap-layer luminescence. We interpret the measured four dark spots as being due to nonradiative recombination. This interpretation is consistent with Fig. 3(c), which shows the corresponding image of the thick lower $\mathrm{ZnMgSSe}$ layer. The projection of the four Frank partial dislocations shown in Fig. 1 onto the $x y$ plane results in a cross structure with reduced photoluminescence. The quantum-well luminescence at the spectral position of the homogeneous quantum-well peak has yet a different structure [Fig. 3(b)]. It exhibits oval-shaped minima at the edges of the bow-tie while the total quantum-well luminescence [Fig. 3(d)] shows bright spots there. This indicates a shift of the photoluminescence towards lower photon ener- gies. This is consistent with the low-photon-energy cut of the quantum-well luminescence, Fig. 3(e) (corresponding to a thickening of the quantum well of one bilayer), which shows oval-shaped maxima where Fig. 3(b) shows oval-shaped minima. On the very low-photon-energy end, Fig. 3(f) (corresponding to a thickening of the quantum well of about 12 bilayers), only the lowest-energy optical transitions contribute. The measured spatial full width at half maximum of the resulting localized exciton emission is $200 \mathrm{~nm}$.

Finally, the approach curves to point B (shown as an inset in Fig. 2) reveal a rise of photoluminescence intensity as the vertical distance between the fiber tip and the sample, $z$, approaches $z=0$. This is indicative of an optical near-field response. (a) and (b) have been measured simultaneously. Note also that this rise is very much steeper when detecting the cap-layer photoluminescence, curve (a) in the inset of Fig. 2, as compared to the quantum-well luminescence, see curve (b) in the inset of Fig. 2.

In conclusion, our low-temperature near-field photoluminescence images of a $\mathrm{ZnSe}$ single-quantum-well structure are an example of how much additional information one can obtain from optical spectroscopy. The optical images reveal that the local quantum-well thickness defines potential minima for electrons and holes at the edges of the bow-tie defect, which leads to a spectral shift and an enhancement of the quantum-well photoluminescence intensity. This local thickening of the quantum well is related to the surface topography seen by AFM, the detailed shapes, however, are different. Only in the $\mathrm{ZnMgSSe}$ barriers, is the nonradiative recombination associated with the four Frank partial dislocations the dominant effect. The size of the bow-tie is expected to scale with the thickness of the lower $\mathrm{ZnMgSSe}$ barrier. Reducing this layer thickness by an order of magnitude might lead to lateral double-well potentials on a $100 \mathrm{~nm}$ scale.

This research has been supported by the DFGSonderforschungsbereich 195 and the DFGGraduiertenkolleg 284, and is performed within the Institut für Nanotechnologie der Universität Karlsruhe (TH).

${ }^{1}$ D. Lüerßen, R. Bleher, H. Richter, Th. Schimmel, and H. Kalt, Appl. Phys. Lett. 75, 3944 (1999).

${ }^{2}$ G. C. Hua, N. Otsuka, D. C. Grillo, Y. Fan, J. Han, M. D. Rigle, R. L. Gunshor, M. Hovinen, and A. V. Nurmikko, Appl. Phys. Lett. 65, 1331 (1994).

${ }^{3}$ L. H. Kuo, K. Kimura, A. Ohtake, S. Miwa, T. Yasuda, and T. Yao, J. Vac. Sci. Technol. B 15, 1241 (1997).

${ }^{4}$ Ch. Adelmann, J. Hetzler, G. Scheiber, Th. Schimmel, M. Wegener, H. B. Weber, and H. v. Löhneysen, Appl. Phys. Lett. 74, 179 (1999).

${ }^{5}$ K.-B. Song, J.-E. Bae, K. Cho, S.-Y. Yim, and S.-H. Park, Appl. Phys. Lett. 73, 2260 (1998).

${ }^{6}$ D. Pahlke, F. Poser, E. Steimetz, M. Pristovsek, N. Esser, and W. Richter, Phys. Status Solidi A 170, 401 (1998).

${ }^{7}$ J. Tanimura, O. Wada, T. Ogama, Y. Endoh, and M. Imaizumi, J. Appl. Phys. 77, 6223 (1995). 